\title{
Hemorragia variceal: profilaxis primaria, secundaria, ligadura, TIPS, prótesis, cianoacrilato, USE, minicoils
}

\author{
Gerardo Alfonso Morales-Fuentes* \\ Servicio de Gastroenterología, Hospital Ángeles Acoxpa; Servicio de Gastroenterología, Hospital Español de México. Ciudad de México, México
}

\begin{abstract}
Resumen:
La hemorragia variceal representa uno de los grandes temas endoscópicos actualmente. En la DDW no se presentaron muchas actualizaciones en cuanto a su profilaxis primaria, pero en cuanto a la terapéutica empleada se le está dando cada vez un mayor peso a la técnica endosvacular de obliteración transvenosa retrógrada con balón oclusivo y al uso de minicoils. Hubo varios estudios demostrando la superioridad de estas técnicas, y la novedad de combinar técnicas conocidas y utilizadas ampliamente como la inyección de cianoacrilato (u otras sustancias) con minicoils y técnicas endovasculares. Los estudios aún cuentan con pocos pacientes pero las expectativas son altas y los resultados obtenidos al momento, tanto en el ámbito clínico como económico, han sido alentadores.
\end{abstract}

Palabras clave: Hemorragia variceal. Várices esofágicas. Várices gástricas. Ligadura variceal. Escleroterapia. Coils. Cianoacrilato. TIPS. BRTO. Cirrosis hepática. Profilaxis primaria. Profilaxis secundaria.

La hemorragia variceal (HV) es una de las mayores complicaciones de la hipertensión portal causada por la cirrosis hepática $(\mathrm{CH})$, corresponde una de las principales causas de hospitalización y muerte en los pacientes cirróticos. Actualmente existen guías de manejo, pero no son del todo seguidas. En el Hospital de la Universidad de Texas ${ }^{1}$ reportan que, de los pacientes con sangrado de tubo digestivo alto en pacientes cirróticos, el 69\% recibió ligadura variceal (LV), pero solo el $65 \%$ egresó con profilaxis secundaria (PS) con betabloqueadores (BB) y solo $27 \%$ tuvieron seguimiento endoscópico en el siguiente mes. Lo que puede traducir la falta de seguimiento a las guías en otros lugares.

En el rubro de la profilaxis primaria, en Egipto ${ }^{2}$ utilizaron la elastografía esplénica para verificar la eficacia dl carvedilol (6.25 mg dos veces/día) como profilaxis primaria, tomando medidas antes y después de 3 meses del uso del BB y verificando con endoscopia, en pacientes conocidos con $\mathrm{CH}$ y VE; dan como corte los $59.85 \mathrm{kPa}$, con S $80 \%$ y E $70 \%$ para determinar la presencia de várices esofágicas (VE), sin que sea predictor de su tamaño ni probabilidad de HV.

La HV es una verdadera urgencia y el tiempo óptimo para realizar la endoscopia es desconocido aún, las guías recomiendan realizarla en las primeras $12-24 \mathrm{~h}$ sin haber uniformidad. Un estudio llevado a cabo en 34 centros de Europa y Canadá ${ }^{3}$ durante casi 4 años, con 1373 pacientes, encontraron que no hay ventaja en la reducción de la mortalidad a 42 días con realizarla a $<6 \quad 0<12 \mathrm{~h}$ con hacerla dentro de las primeras $24 \mathrm{~h}$ mientras que la mortalidad sí se incrementa al realizarla después de las $24 \mathrm{~h}$ y la asociación es mayor si el Child-Pugh es mayor.

Existen escalas predictoras que se pueden realizar en la valoración inicial: Rassameehiran ${ }^{4}$ comparó

\section{Correspondencia:}


varias escalas: Glasgow-Blatchford, pre-Rockall, Rockall, AIMS65, MELD, MELD-Na y Shock index, donde concluye que un Shock index $>0.85$ tiene mayor probabilidad de requerir LV y Hui ${ }^{5}$ concluye que la escala AIMS65 es superior y las más sencilla en comparación con las demás para predecir la mortalidad dentro de las primeras 6 semanas.

Dentro del tratamiento de la HV, se presentaron 2 trabajos referentes al uso de polvos hemostáticos, en uno de ellos con 93 pacientes $^{6}$, en solo el $5 \%$ de los casos fue utilizado en casos de VE sin mayores especificaciones y en el metaanálisis de 52 estudios hecho por Tavares ${ }^{7}$, si incluye 5 estudios donde concluyen un éxito de hemostasis de $90.4 \%$ y $4.2 \%$ de resangrado.

En cuanto a la profilaxis secundaria, para el uso de TIPS se presentó un metaanálisis de Paleti $^{8}$ donde comparaba dos tratamientos endovasculares: TIPS y obliteración transvenosa retrógrada con balón oclusivo (BRTO), para várices gástricas (VG) en 8 estudios con 741 pacientes, donde ambas técnicas tienen tasas similares de éxito y hemostasis, pero el BRTO se asoció a menos resangrado y encefalopatía postquirúrgica. Otro enfoque del tratamiento angiográfico es el que ofrece Bazerbachi ${ }^{9}$, donde en 30 pacientes candidatos para inyección de cianoacrilato (cyan) en las VG lo hace previa colocación de un balón oclusivo endovascular en los shunt gastrorrenales, culpables de los efectos embólicos adversos de esta técnica, y concluye que en $78 \%$ de los casos hubo erradicación de las VG y solo $10 \%$ de complicaciones, entre ellas el embolismo. Continuando con técnicas endovasculares, en China, Luo $^{10}$ comparó dos grupos, uno en el que el tratamiento de las VG era con BRTO y otro con cyan, fue un grupo de 32 pacientes en poco menos de 2 años, el éxito de la técnica fue de $100 \%$ para ambos, el resangrado fue de $3 \%$ para el BRTO y $31 \%$ para el cyan, a 2 años la probabilidad de estar libre de sangrado fue de $94.7 \%$ para el BRTO y $66.9 \%$ para el cyan, la supervivencia a 2 años fue similar; concluyendo la superioridad del BRTO sobre el cyan. Por medio de la cápsula endoscópica, se han identificado algunos efectos en los pacientes que se someten a escleroterapia de las várices y estos son que hasta el $90 \%$ de los pacientes presentan exacerbación de la enteropatía portal hipertensiva ${ }^{11}$, demostrada por el edema de las vellosidades intestinales antes y después del tratamiento, sin embargo, no se ha ligado a ningún escenario clínico.

El ultrasonido endoscópico (USE) se utiliza para determinar el mejor punto para aplicar cierto tratamiento, ya que la inyección a ciegas de los pegamentos tisulares puede incrementar la incidencia de complicaciones. Un primer metaanálisis americano ${ }^{12}$ revisado, incluyó 6 estudios, con un total de 340 pacientes con VG, donde se utiliza al USE para guiar al endoscopista y dirigir el tratamiento angiológico (ya sea escleroterapia con cyan o inyección de coils) obteniendo un éxito clínico en $87 \%$, con resangrado en $12.57 \%$ y un $10.45 \%$ de complicaciones. Chen ${ }^{13}$, en China, realizó un estudio con 69 pacientes divididos en dos grupos, uno en el que la inyección era guiada por USE, es decir, se realizaba una devascularización variceal guiada de las colaterales más importantes y el otro grupo se inyectaba cyan sin USE, es decir a ciegas, los pacientes fueron seguidos mensualmente y en caso necesario se repetía el procedimiento hasta lograr la completa obliteración de las VG o que hubiera una HV mayor, concluyó que el grupo donde no se utilizó USE tuvo una mayor probabilidad de tener un sangrado masivo y pérdida de al menos $3 \mathrm{gr} / \mathrm{dl}$ de hemoglobina que en el grupo donde se utilizó USE, y que la cantidad de pegamento tisular inyectado y el número de inyecciones fue menor al utilizar el USE, y no tuvo complicaciones de ningún tipo.

En cuanto al uso de coils para el tratamiento de las VG se ha propuesto su inyección junto con ciertas sustancias gelatinosas esponjosas absorbibles (gelfoam) y se ha comprobado su utilidad contra la inyección del cyan. Así tenemos el estudio realizado por Bazarbashi en Boston en 2018, donde a un grupo con 10 pacientes $^{14}$ los cuales inyectó coils con gelfoam guiado por USE (grupo A) y posteriormente los comparó con otro grupo de 30 pacientes a los cuales inyectó solo cyan ${ }^{15}$ (grupo B), el éxito de la técnica en $A$ fue de $100 \%$ (aunque un paciente tuvo persistencia del shunt gastrorrenal, por lo que solo se inyectaron coils) y del B fue de $96.7 \%$, las complicaciones inmediatas en A fue de $10 \%$ (dolor abdominal, no hubo reportes de embolismos) y las de $B$ fueron de $20 \%$, el $0 \%$ del A requirió reintervención en el seguimiento a los 6 meses y $56 \%$ la requirió en el $B$ y la mortalidad a los 6 meses fue de $10 \%$ en A y $16.7 \%$ en $\mathrm{B}$. El mismo equipo presentó un metaanálisis de 11 estudios (536 pacientes) comparando tres tratamientos guiados por USE para VG, ya que es claro que la guía por USE tiene ventajas: cyan (A), coil+cyan (B) y coil solos (C). El éxito técnico se alcanzó en los tres, el éxito clínico más bajo $(90 \%)$ fue en $\mathrm{C}$, los mayores efectos adversos fueron con $\mathrm{A}$ $(21 \%)$ y los menores con $C(3 \%)$, la tasa de reintervención más baja (15\%) fue en $B$, mientras que en $A$ 
y C fue de $25 \%$, finalmente la tasa de resangrado más baja se logró con B (14\%), seguida de C (17\%) y la más alta fue de $\mathrm{A}(30 \%)$. Con lo anterior se concluye que la combinación de coil+cyan es la mejor estrategia terapéutica para las $\mathrm{VG}^{16}$.

Hubo dos metaanálisis que compararon los métodos endoscópicos (ligadura y cyan) y los endovasculares (BRTO y TIPS): el primero de ellos de 20 estudios con 1175 pacientes $^{17}$ concluye que el BRTO es la mejor modalidad para el tratamiento de VG en cuanto a menor mortalidad y resangrado, seguido por el cyan, TIPS que tiene mayor riesgo de encefalopatía y la ligadura siendo el método que menos ventajas aporta (se debe notar que no se incluyeron los coils solos o con gelfoam en este estudio). El otro metaanálisis de 98 pacientes ${ }^{18}$ del equipo de Bazarbashi en Boston compara los métodos endoscópicos (cyan y coils) y los endovasculares (BRTO y TIPS), siendo la serie más grande donde si se incluyen los coils en comparación con los métodos radiológicos, concluye que precisamente los coils son la mejor opción en cuanto a que tuvieron $0 \%$ de necesidad de reintervención a los 6 meses y siendo los menores con resangrado. Este mismo equipo hace una comparación acerca de los costos de las mismas opciones terapéuticas endoscópicas contras las endovasculares, concluyendo que las opciones endoscópicas son hasta 6 veces más económicas que las endovasculares, principalmente los coils, tanto en el costo económico de la técnica en sí, como en su seguimiento ${ }^{19}$.

En el rubro de misceláneos en relación al manejo en general de los pacientes con $\mathrm{CH}$, un estudio concluye que el agregar una dieta enteral intrahospitalaria rica en aminoácidos de cadena ramificada mejora la función hepática y el estado general de los pacientes ${ }^{20}$, y otro estudio que la presencia de Helicobacter pylori se asocia a un menor riesgo de resangrado en pacientes con VE en pacientes con Child-Pugh $A^{21}$. No hubo información nueva o estudios del empleo de prótesis para el control de la HV o su profilaxis, solo fueron comentadas como herramientas actuales, pero queda claro que las nuevas técnicas superan por mucho su empleo.

\section{Conflicto de intereses}

No existen conflictos de interés. Sin patrocinio de la industria.

\section{Bibliografía}

1. Chatila A, Bilal M, Kassem A, et al. Evaluating and improving quality of care in patients with end-stage liver disease. DDW 2019, San Diego, USA. Su1489.

2. Khorshid M, Eisharkawy A, Hamza I, et al. Measurement of splenic stiffness in the prediction of esophageal varices and the response to carvedilol therapy in portal hypertensive patients. DDW 2019, San Diego, USA. Mo1529.

3. Laursen S, Stanley A, Hernández V, et al. Optimal timing of endoscopy is associated with lower 42-day mortality in variceal bleeding. DDW 2019 , San Diego, USA. Session number 4175.

4. Rassameehiran S, Tetangco E, Tan G, et al. Predictor of endoscopic variceal ligation for cirrhotic patients presenting with UGIB. DDW 2019, San Diego, USA. Sa1240.

5. Hui S, Chung W, McNamara L, et al. AIMS65, a risk stratification score for upper gastrointestinal bleeding, accurately predicts mortality in patients presenting with acute variceal bleeding: a prospective multicenter study. DDW 2019, San Diego, USA. Sa1244.

6. Chahal D, Ali N, Donnellan F. Clinical outcomes of hemospray use in upper \& lower gastrointestinal bleeding. DDW 2019, San Diego, USA. Tu1119.

7. Tavares D, Marques W, Ottoboni V, et al. The use of hemostatic powder in upper gastrointestinal bleeding: a systematic review and meta-analysis. DDW 2019, San Diego, USA. Tu1094.

8. Paleti S, Nutalapati V, Fathallan J, et al. Balloon-occluded retrograde transvenous obliteration (BRTO) versus transjugular intrahepatic portosystemic shunt (TIPS) for treatment of gastric varices due to portal hypertension: a systematic review and meta-analysis. DDW 2019, San Diego, USA. Session number 4275.

9. Bazerbachi F, Dobashi A, Kumar S, et al. Safety and efficacy of combined endoscopic cyanoacrylate injection and angiographic balloon catheter occlusion of gastrorenal shunts in patients with bleeding gastric varices. DDW 2019, San Diego, USA. Su1535.

10. Luo X, Wang X, Yang $L$, et al. Randomized trial of balloon-occluded retrograde transvenous obliteration versus cyanoacrylate injection for prevention of gastric variceal rebleeding. DDW 2019, San Diego, USA. Session number 3280

11. Otani I, Oka S, Tanaka S, et al. Endoscopic injection sclerotherapy for esophageal varices exacerbates portal hypertensive enteropathy. DDW 2019, San Diego, USA. Su1323.

12. Koroglu E, Shrivastava A, Akyuz U, et al. EUS guided angiotheraphy is highly effective to treatment of gastric varices: a systematic review and meta-analysis. DDW 2019, San Diego, USA. Su1232.

13. Chen $\mathrm{H}$, Wang $\mathrm{Y}$, Jiang $\mathrm{L}$, et al. A randomized controlled trial of endoscopic ultrasound guided selective variceal devascularization versus standard endoscopic variceal obturation for secondary prophylaxis of gastroesophageal variceal bleeding in a tertiary teaching hospital. DDW 2019, San Diego, USA. Session number 5255

14. Bazarbashi A, Wang T, Ge P, et al. Endoscopic ultrasound-guided treatment of gastric varices with coil embolization and absorbable gelatin sponge: a novel alternative to cyanoacrylate. DDW 2019, San Diego, USA.

15. Bazarbashi A, Wang T, Hathorn $\mathrm{K}$, et al. For treatment of gastric varices, EUS-guided coil embolization leads to lower transfusion requirements and re-intervention rates compared to traditional cyanoacrylate injection: a matched cohort study. DDW 2019, San Diego, USA. Su1200.

16. Bazarbashi A, McCarty T, Hathorn K, et al. Combination therapy vs monotherapy for EUS-guided management of gastric varices: a systematic review and meta-analysis. DDW 2019, San Diego, USA. Su1521.

17. Khan M, Iqbar U, Ryou Z, et al. What is the best therapeutic modality for managing gastric varices? A systematic review and network meta-analysis to compare glue injection, TIPS, BRTO and banding. DDW 2019, San Diego, USA. Mo1525.

18. Wang T, Bazarbashi A, Ge P, et al. Comparison of endoscopic and IR treatments of gastric varices: EUS-guided coil therapy has decreased re-intervention and re-admission rates compared to endoscopic cyanoacrylate injection, BRTO, and TIPS. DDW 2019, San Diego, USA. Mo1528.

19. Wang T, Bazarbashi A, Hathorn K, et al. EUS-guided coil therapy is cost favorable over IR-guided therapies for treatment of gastric variceal bleeding: a cost utility analysis. DDW 2019, San Diego, USA. Su1203.

20. Kojima Y, Takeuchi T, Nishida S, et al. Usefullness of branched-chain amino acid-enriched enteral nutrition in the endoscopic treatment of esophageal varices. DDW 2019, San Diego, USA. Su2029.

21. Kate V, Sureshkumar S, Varuna S, et al. Association between variceal bleed and Helicobacter pylori infection in patients of cirrhosis with portal hypertension - A cohort study. DDW 2019, San Diego, USA. Su 1538. 